\title{
Direct Analysis of the Current Density vs. Voltage Curves of a CdTe Module during Outdoor Exposure
}

\author{
Carolin Ulbrich $^{\mathrm{a}, 1}$, Dirk C. Jordan ${ }^{\mathrm{b}}$, Sarah R. Kurtz ${ }^{\mathrm{b}}$, Andreas Gerber ${ }^{\mathrm{a}}$, Uwe Rau ${ }^{\mathrm{a}}$ \\ ${ }^{a}$ IEK5-Photovoltaik, Forschungszentrum Jülich GmbH, Wilhelm-Johnen-Straße, 52428 Jülich, Germany \\ ${ }^{\mathrm{b}}$ National Renewable Energy Laboratory, 15013 Denver West Pkwy., Golden, CO, 80401, USA
}

\begin{abstract}
Current density-voltage $(J V)$ curves of a CdTe photovoltaic module measured during 2.5 years of outdoor operation are analyzed using a phenomenological four-parameter equation. This approach is shown to be more accurate than fitting the same data set to the conventional five parameter diode equation. The four extracted parameters are the short-circuit current density $J_{\mathrm{sc}}$, open-circuit voltage $V_{\mathrm{oc}}$, and the differential resistances $R_{\mathrm{sc}}$ and $R_{\mathrm{oc}}$, i.e., the reciprocal slopes of the $J V$ curves at short circuit and open circuit, respectively. The dependencies of all four parameters on module temperature and irradiation intensities are analyzed in terms of physically motivated models. The coefficients derived from these models are then used to transform the photovoltaic parameters to reference conditions and to investigate the degradation of the module. The models for $R_{\mathrm{sc}}$ and $R_{\mathrm{oc}}$ involve voltage-dependent carrier collection. This feature appears prominent already in the initial $J V$ curves, but becomes even more dominant with increasing exposure time. Voltage-dependent carrier collection also explains that the diode ideality factor determined from $V_{\mathrm{oc}}$ vs. $J_{\mathrm{sc}}$ curves is different from that determined using $R_{\mathrm{oc}}$ vs. $1 / J_{\mathrm{sc}}$ plots. The degradation of the module is essentially reflected in fill factor losses caused by a decrease of $R_{\mathrm{sc}}$ and an increase of $R_{\mathrm{oc}}$. Additionally, the $V_{\mathrm{oc}}$ vs. $\log \left(J_{\mathrm{sc}}\right)$ curves of the module behavior at low irradiation unveil an increasing influence of a double-exponential diode behavior.
\end{abstract}

Keywords - current-voltage characteristic, degradation, monitoring, CdTe, diode ideality factor, reliability.

\section{Introduction}

In view of a global solar photovoltaic (PV) capacity surpassing the $100 \mathrm{GW}$ milestone [EPIA, 2014], accurate monitoring of the outdoor performance of solar modules or PV systems is of utmost importance. Continuous measurement of complete current density-voltage $(J V)$ curves of modules during outdoor operation yields especially important information on specific technologies. The evaluation of the $J V$ curve data requires the application of specific models, as reviewed recently by Cotfas et al., 2013. Most performance models account for the varying irradiance and module temperature during operation [King et al., 2004; Williams et al., 2005; Guérin de Montgareuil, 2007; Sutterlueti et al., 2011; Merten et al., 2012; Stein et al., 2013]. The coefficients derived by application of the models to the data are used in the analysis of the outdoor degradation, but also, to predict the performance of the module under given irradiation conditions, temperatures, and other conditions. Failure of accurate prediction is an indicator of additional degradation, i.e., a change of the physical module behavior over time of exposure. When considering time intervals shorter than the entire outdoor exposure, specific changes of the module coefficients with time and (changing) dependence on irradiance and temperature can be calculated and the effect of their degradation is quantifiable. The translation of acquired outdoor data to standard conditions [IEC 60891] requires the determination of temperature-correction and curve-correction parameters that are often measured indoors and usually only at the beginning of the exposure [IEC60904]. These coefficients may not hold for the

${ }^{1}$ corresponding author, contact: c.ulbrich@fz-juelich.de, +492461616310

Non-standard abbreviations: KH: equation proposed by Karmalkar and Haneefa (2008)

Email addresses: dirk.jordan@nrel.gov, sarah.kurtz@nrel.gov, a.gerber@fz-juelich.de, u.rau@fz-juelich.de 
entire time of outdoor exposure. Additionally, coefficients determined for many performance models are not physically motivated and the corresponding changes over operation time cannot be interpreted in terms of changing physical device properties.

The present paper introduces a simple four-step procedure to analyze acquired $J V$ data in terms of the underlying physics. In a first step (i), all measured $J V$ curves are fitted with the help of a four-parameter equation [Karmalkar and Haneefa, 2008]. The four independent parameters represent or translate into the short-circuit current density $J_{\mathrm{sc}}$, open-circuit voltage $V_{\mathrm{oc}}$, and differential resistances $R_{\mathrm{sc}}$ and $R_{\mathrm{oc}}$ (i.e., the reciprocal slopes of the $J V$ curves) at short circuit and open circuit. The use of the Karmalkar-Haneefa (KH) equation allows us to reduce the number of parameters because the fits obtained using only $J_{\mathrm{sc}}, V_{\mathrm{oc}}, R_{\mathrm{sc}}$, and $R_{\text {oc }}$ determine the entire $J V$ curve including the fill factor $F F$. In step (ii), we analyze the dependence of $J_{\text {sc }}$ on module temperature $T$ and irradiation intensities $\phi$, as well as the dependencies of the other parameters on $T$ and $J_{\text {sc }}$. This analysis yields physically motivated coefficients for the functional dependence of the four parameters on $T$ and $\phi$. A period of one to two weeks with acquisition of $J V$ curves every 15 min is sufficient for a reliable determination of these coefficients. Note that the number of data points is reduced by an applied filtering before determination of the coefficients. Step (iii) uses the model equation and the acquired parameters to calculate the PV output parameters at reference conditions. Finally, in step (iv), the time series of the PV output parameters, as well as that of the time development of the coefficients, is analyzed to investigate the degradation. In the present case, the analysis unveils clear fingerprints of an increasing influence of voltage-dependent carrier collection. Whereas the accuracy of our method is sufficient to analyze the degradation, a correction of the absolute values of the maximum output power is necessary to improve the precision of absolute output power prediction to the usual standard.

\section{Experimental Field Data}

The investigated CdTe module is a prototype chosen because it shows an unusually high degradation rate, allowing us to better study the signature of degradation. The module consisted of a series connection of 58 cells with a cell area of $8675.4 \mathrm{~cm}^{2}$. The module was exposed outdoors on the Performance and Energy Rating Testbed (PERT) at the National Renewable Energy Laboratory (NREL), Golden, CO, USA. The module was mounted at the latitude tilt of $40^{\circ}\left( \pm 1^{\circ}\right)$ facing south $\left( \pm 2^{\circ}\right)$ and was held at maximum power with current-voltage curves taken typically every 15 minutes. Environmental parameters were recorded simultaneously and included plane-of-array (POA) irradiance ( $\mathrm{Wm}^{-2}$, measured by a thermopile), wind speed $(\mathrm{m} / \mathrm{s})$, ambient temperature $\left({ }^{\circ} \mathrm{C}\right)$, and back-of-module temperature $\left({ }^{\circ} \mathrm{C}\right)$, as previously detailed by del Cueto (2005). For the analysis, we only used the raw current-voltage $I V$ curve data, as well as the temperature $T_{\text {meas }}$ measured at the rear of the module and the POA irradiance $\phi_{\text {meas }}$. Modules were not cleaned. The irradiance sensor was cleaned weekly. Measurements on a nearby pair of cleaned and uncleaned modules showed that average soiling was $<2 \%$. The related noise is effectively removed during the analysis, as described below.

The data filtering follows the approach published by Jordan and Kurtz (2014). We sorted out data measured under unstable irradiation conditions (irradiance change $>5 \%$ between recorded value before and after each trace) and at module temperatures lower than $5^{\circ} \mathrm{C}$. Also, curves with unreasonably low or high fill factor $F F$ or $I_{\text {sc,meas }} / \phi_{\text {meas }}$ values were carefully removed.

A reversible degradation is typical for thin-film devices. CdTe modules are built in p-n configuration. Instead of doped layers of the same material, a CdS layer forms the n-type buffer for a CdTe p-layer. The 
Ohmic contact metallization is deposited last. Sometimes, a Cu-containing component is included that can cause instabilities [Gostein and Dunn, 2011]. There are several further sources of degradation, for example, trap states [Sasala and Sites, 1993] in the absorber junction, series resistance of the back contact [Carlsson and Brinkman, 2006], and ion migration [Pudov et al., 2002; Jenkins et al., 2003; Corwine et al., 2004]. Light-exposure and bias application can enhance or deteriorate device performance depending on conditions and specific arrangement of the layers, material composition, and the back contact [Carlsson and Brinkman, 2006; del Cueto and von Roedern, 2006; Hegedus and McCandless, 2005] in CdTe modules. It is impossible to distinguish between those degradation mechanisms looking at the $J V$ data alone. Yet, the $J V$ data enable us to investigate the signature of these degradation mechanisms. In the following, we show how such signatures are distilled out of the $J V$ data acquired during outdoor exposure.

\section{Analysis Method}

In 2008, Karmalkar and Haneefa proposed a phenomenological equation for evaluating illuminated $J V$ curves of solar cells according to

$$
J(V)=J_{\mathrm{sc}}\left[(1-\gamma) \frac{V}{V_{\mathrm{oc}}}+\gamma\left(\frac{V}{V_{\mathrm{oc}}}\right)^{m}-1\right]
$$

Compared to the many existing methods to analyze $J V$ curves [Cotfas et al., 2013], Eq. 1 has the advantage that it only depends on four parameters, i.e., short-circuit current density $J_{\text {sc }}$, open-circuit voltage $V_{\text {oc }}$, and the dimensionless quantities $\gamma$ and $m$. This equation also has the advantage of being very easy to implement since all four parameters can be directly derived from the curve, then optimized (to reduce the effects of noise or inaccurate initial estimation of the four parameters) quite easily with a regression relative to the power vs. voltage curve. Illuminated current-voltage curves from a wide variety of solar cells can be fitted accurately by this approach. Equation 1 by itself has no physical meaning; but various, more complex, physically motivated solar cell models are compatible with the equation, as shown by Das and Karmalkar (2011).

From Eq. 1, the fill factor $F F$ can be calculated via

$$
F F=\frac{V_{\mathrm{mpp}}}{V_{\mathrm{oc}}}\left\{1-(1-\gamma) \frac{V_{\mathrm{mpp}}}{V_{\mathrm{oc}}}-\gamma\left(\frac{V_{\mathrm{mpp}}}{V_{\mathrm{oc}}}\right)^{m}\right\},
$$

where the voltage $V_{\mathrm{mpp}}$ at the maximum power point is given by [Das and Karmalkar, 2011]

$$
\frac{V_{\mathrm{mpp}}}{V_{\mathrm{oc}}}=(m+1)^{-1 / m}-\left[(m+1)^{-1 / m}-0.5\right](1-\gamma)^{1.58+0.05 m}
$$

and the current density at the maximum power point $J_{\mathrm{mpp}}=J_{\mathrm{mpp}}\left(V_{\mathrm{mpp}}\right)$ is obtained by Eq. 1 . For the present purpose, we make use of the fact that the validity range of Eq. 1 - namely, the quadrant of energy extraction of the illuminated characteristics - fits to the range of our experimental data. As shown below, Eq. 1 is able to fit the relatively featureless current-voltage curves of PV modules over a wide range of irradiation intensities $\phi$ and module temperatures $T$. 
Furthermore, the parameters $\gamma$ and $m$ are directly related to the differential resistances $R_{\mathrm{oc}}$ and $R_{\mathrm{sc}}$, respectively, the differential conductances $G_{\mathrm{oc}}$ and $G_{\mathrm{sc}}$ at the short-circuit (sc) and open-circuit (oc) points of the current-voltage curves via

and

$$
\frac{1}{R_{\mathrm{sc}}}=G_{\mathrm{sc}}=\left.\frac{d J}{d V}\right|_{V=0}=\frac{J_{\mathrm{sc}}}{V_{\mathrm{oc}}}(1-\gamma)
$$

$$
\frac{1}{R_{\mathrm{oc}}}=G_{\mathrm{oc}}=\left.\frac{d J}{d V}\right|_{V=V_{\mathrm{oc}}}=\frac{J_{\mathrm{sc}}}{V_{\mathrm{oc}}}(1-\gamma+\gamma m) .
$$

Inversion of Eqs. 4 and 5 yields

$$
\gamma=1-G_{\mathrm{sc}} \frac{V_{\mathrm{oc}}}{J_{\mathrm{sc}}}
$$

and

$$
m=\frac{V_{\mathrm{oc}}\left(G_{\mathrm{oc}}-G_{\mathrm{sc}}\right)}{J_{\mathrm{sc}}-V_{\mathrm{oc}} G_{\mathrm{sc}}} .
$$

We note that the quantities $R_{\mathrm{sc}}$ and $R_{\mathrm{oc}}$ are not identical to the parallel and series resistance $R_{\mathrm{p}}$ and $R_{\mathrm{s}}$ of the textbook equivalent circuit of a solar cell. However, possible changes in $R_{\mathrm{sc}}$ and $R_{\mathrm{oc}}$ give an indication of whether a degradation mechanism is more parallel-resistance-like (indicated by changes in $R_{\mathrm{sc}}$ ) or more series-resistance-like (indicated by changes in $R_{\mathrm{oc}}$ ). Furthermore, both quantities can be described in terms of physical models, as shown in sections 4.2 and 4.4.

Figures $1 \mathrm{a}$ and $1 \mathrm{~b}$ display various measured and fitted $J V$ curves, along with the graphical definitions of the four parameters (Fig. 1a) used in the $\mathrm{KH}$ equation. Note here and in the following that the data are scaled to the current density $J$ per unit cell area and the voltage $V$ per cell. With Eq. 1, we are able to fit the $J V$ curves of PV modules over a wide range of irradiation intensities $\phi_{\text {meas }}$ and temperatures $T_{\text {meas }}$. 


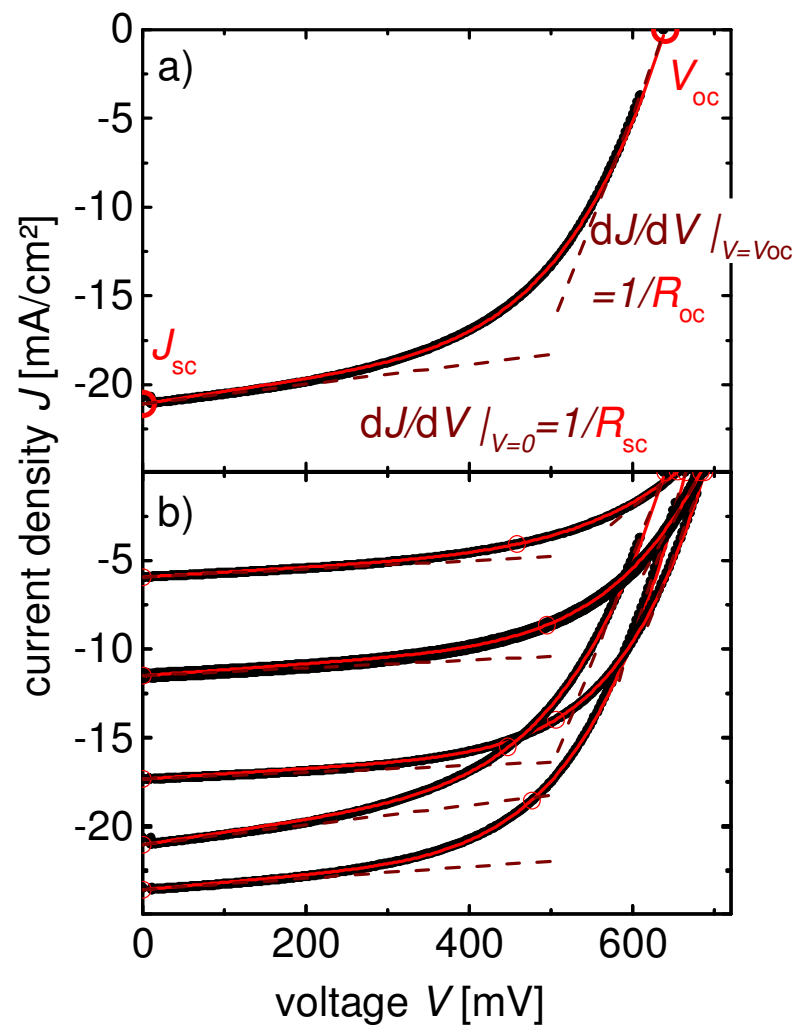

Fig. 1. Fit of the Karmalkar-Haneefa (KH) equation to the measured JV curves. a) The KH equation uses four fit parameters: $J_{\mathrm{sc}}$ open-circuit voltage $V_{\mathrm{oc}}$ and differential resistances $R_{\mathrm{sc}}$ and $R_{\mathrm{oc}}$ as marked. $b$ ) The equation provides very good fits of the JV curves (raw data in black) at various stages in degradation and acquired at various irradiance levels (red lines).

\section{Irradiance and Temperature Dependence}

As mentioned previously (step (ii) in section 1), we analyze all four parameters $J_{\mathrm{sc}}, V_{\mathrm{oc}}, R_{\mathrm{sc}}$, and $R_{\mathrm{oc}}$ used in the KH equation in terms of their dependencies on module temperature $T$ and irradiation intensities $\phi\left(J_{\mathrm{sc}}\right)$ or in terms of their dependencies on module temperature and short-circuit current density $J_{\mathrm{sc}}\left(V_{\mathrm{oc}}, R_{\mathrm{sc}}, R_{\mathrm{oc}}\right)$.

\subsection{Short-Circuit Current Density}

The short-circuit current density $J_{\mathrm{sc}}$ is proportional to the irradiation intensity $\phi_{\text {meas }}$, where the proportionality constant depends on the module temperatures $T_{\text {meas }}$ according to

$$
J_{s c, \text { meas }}\left(\phi_{\text {meas }}, T_{\text {meas }}\right)=\left\{\alpha_{\mathrm{Jsc}} T_{\text {meas }}+\kappa_{\mathrm{Jsc}}\right\}_{\phi_{\text {meas }}} .
$$

Knowledge of the coefficients $\alpha_{\mathrm{Jsc}}$ and $\kappa_{\mathrm{Jsc}}$ allows one to calculate $J_{\text {sc } \mathrm{ref}}$ for any desired reference condition expressed by a reference irradiance $\phi_{\text {ref }}$ and a reference module temperature $T_{\text {ref }}$. Likewise, one may translate the raw values $J_{\mathrm{sc}}$ directly to the reference condition using

$$
J_{\text {sc@ref }}=\left\{\alpha_{\mathrm{Isc}}\left(T_{\text {ref }}-T_{\text {meas }}\right)\right\} \phi_{\text {ref }}+J_{\text {sc, } \text { meas }}\left(\phi_{\text {ref }} / \phi_{\text {meas }}\right),
$$

where the first term accounts for the correction of $T_{\text {meas }}$ and the second for the corrections of $\phi_{\text {meas }}$.

Figure 2 shows the $J_{\text {sc,meas }}$ data for a one-week time interval versus measured irradiance $\phi_{\text {meas }}$ (raw data are black dots). The raw data are used to calculate the respective coefficients $\alpha_{\mathrm{Jsc}}$ and $\kappa_{\mathrm{Jsc}}$ for temperature and 
irradiance dependence. The derivation of these coefficients from outdoor data include the dependence of the module performance on $T$ and $\phi$ as well as the effects of any systematic variations in spectrum with $T$ or $\phi$, including angle-of-incidence effects. This figure shows the result of a temperature correction only, to $T_{\text {ref }}$ $=40^{\circ} \mathrm{C}$ (using only the first correction term in Eq. 9, orange triangles in figure). The effect of the temperature $T_{\text {meas }}$ is small and the linear dependence of $J_{\text {scmeas }}$ on $\phi_{\text {meas }}$ remains essentially unchanged. The final values $J_{\text {sc@ref }}$ (blue diamonds) were translated to both reference values $\phi_{\text {ref }}=1000 \mathrm{Wm}^{-2}, T_{\text {ref }}=40^{\circ} \mathrm{C}$. The data above $\phi_{\text {meas }}=500 \mathrm{Wm}^{-2}$ show no irradiance dependence after this correction, whereas some scatter of values is observed in the low irradiance range. Note that week 1 is not the first week of outdoor exposure. Some tests were performed indoors and outdoors prior to the long-term data acquisition.

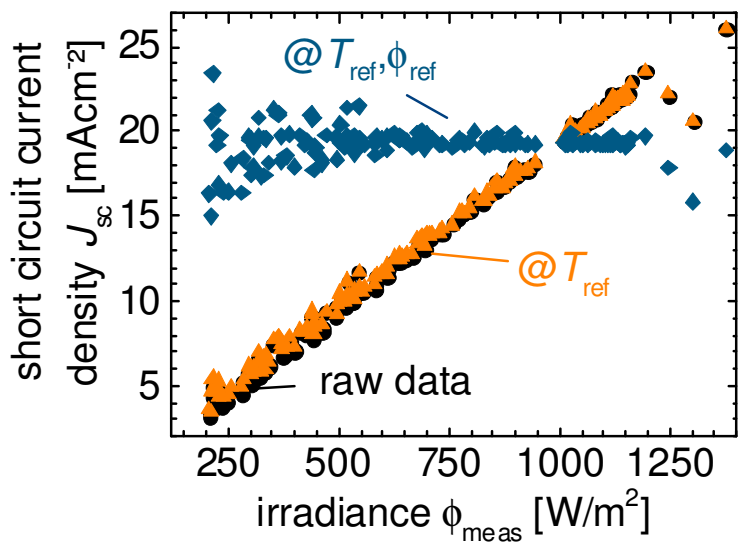

Fig. 2. Translation of short-circuit current density $J_{s c \text { meas. }}$. Data from outdoor-measured JV curves are translated to reference conditions $\phi_{\text {ref }}=1000 \mathrm{Wm}^{-2}, T_{\text {ref }}=40^{\circ} \mathrm{C}$ shown as a function of measured irradiance $\phi_{\text {meas }}$ for week 1. Raw data (black dots) are translated to $J_{\text {sc@ref }}$ at reference module temperature $T_{\text {ref }}$ (orange triangles) and reference irradiation intensity $\phi_{\text {ref }}$ (blue diamonds).

Figure 3 shows the short-circuit current density $J_{\text {sceref }}$ translated to reference conditions based on a weekly data analysis. $J_{\text {sceref }}$ shows a slight seasonal variation, but no apparent degradation over the 2.5 -year time period. The seasonal variation roughly follows the variation in average photon energy (APE) [Jardine et al., 2002], i.e., the variations of the ratio between blue and red photons in the incident spectrum compared to the AM1.5g spectrum. Because no measurements of the local spectrum were acquired at the time of the experiment, a correction of the data for spectral variations is not considered in the following.

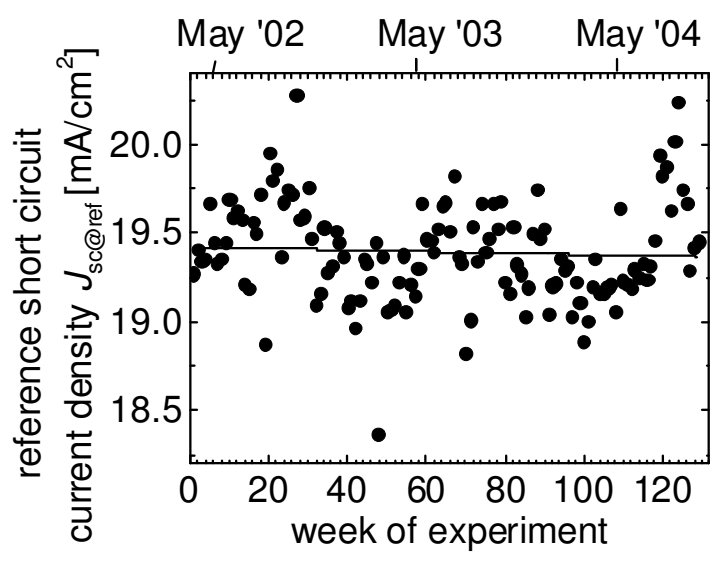

Fig. 3. Short-circuit current density $J_{\text {sc@ref }}$ (black dots) translated to reference module temperature $T_{r e f}=40^{\circ} \mathrm{C}$ and reference irradiation intensity $\phi_{r e f}=1000 \mathrm{Wm}^{-2}$. Except for a small seasonal variation, the 
data show no significant degradation. A linear fit to the data (black line) has a slope of $-0.02( \pm 0.04) \mathrm{mAcm} \mathrm{cm}^{-2} /$ year, i.e., the degradation is below the error limit.

Linear fits are added to Figs. 5a), 5b), 7, 9a), 9b), and 12 (black lines), as done here in Fig. 3. Note also that in the following, we have replaced the dependence on $\phi_{\text {meas }}$ by a dependence on $J_{\text {sc,meas }}$. This replacement is justified by the linear interdependence of both quantities, shown for the first week of data in Fig. 2. The slope of this linearity changes little following the seasonal irradiation changes over time of outdoor exposure (not shown). As a reference value, we replace $\phi_{\text {ref }}=1000 \mathrm{Wm}^{-2}$ by $J_{\text {sc,ref }}=19.3 \mathrm{mAcm}^{-2}$, the value of $J_{\text {sc@ref }}$ in the first week. By substituting $\phi_{\text {meas }}$ by $J_{\text {sc,meas }}$, we eliminate those effects of fluctuations due to the seasonal spectral changes. Annealing and degradation due to temperature and illumination exposure are still reflected by the data. This replacement, following the self-irradiance approach as suggested by King et al., (2004), reduces the scatter in the analysis of the outdoor data.

\subsection{Differential conductance at short circuit}

In an ideal solar cell, one would not expect a dependence of the conductance $G_{\text {sc.meas}}$, or the resistance

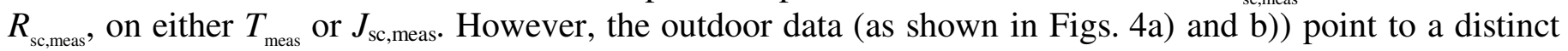
direction. Here, we find a pronounced dependence on both external parameters that is well described by a linear dependence on $T_{\text {meas }}$ and $J_{\text {sc,meas }}$

$$
G_{\mathrm{sc}, \text { meas }}\left(J_{\mathrm{sc}, \text { meas }}, T_{\text {meas }}\right)=G_{\mathrm{sc}, 0}+\alpha_{\mathrm{Gsc}} T_{\text {meas }}+\kappa_{\mathrm{Gsc}} J_{\text {sc, meas }} .
$$

Using Eq. 10, we obtain the translation equation

$$
G_{\mathrm{sc} @ \text { ref }}=G_{\mathrm{sc}, \text { meas }}+\alpha_{\mathrm{Jsc}}\left(T_{\text {ref }}-T_{\text {meas }}\right)+\kappa_{\mathrm{Jsc}}\left(J_{\mathrm{sc}, \text { ref }}-J_{\mathrm{sc}, \text { meas }}\right) .
$$

Note that the strong dependence of the differential resistance $G_{\mathrm{sc}}$ on $J_{\mathrm{sc}}$ can be explained by voltagedependent photocurrent collection (Hegedus et al., 2007), where the photocurrent at a given junction voltage is given by $J_{p h}\left(V_{j}\right)=J_{p h}^{\max } f\left(V_{j}\right)$, where $f\left(V_{j}\right)$ is the collection efficiency or collection function. The coefficient $\kappa_{\text {Gsc }}$ is then given by $\kappa_{\text {Gsc }}=d f / d V_{j} / f$. Note that an illumination-dependent shunt resistance could have the same effect, which would lead to an expression that is mathematically equivalent to Eq. (10) because of the linear dependence of $J_{\text {sc,meas }}$ on $\phi_{\text {meas }}$ (cf. Fig. 2). Furthermore, solar cells - and especially solar modules with a spatially inhomogeneous distribution of series and shunt resistances - may also exhibit such bias-dependent shunting behavior (see, e.g., Breitenstein, 2013, as well as Bowden and Rohatgi, 2001).

Figure 4 presents the raw data of the conductance at short-circuit $G_{\text {scmeas }}$ extracted from the $J V$ curves of one week of outdoor exposure (black dots) for week 1 (Fig. 4a) and week 106 (Fig 4b). Note the change of scale between (a) and (b), reflecting the increase of $G_{\text {sc@ref }}$ during exposure time. The temperature correction alone (orange triangles) has only a weak influence in the low-temperature/low-irradiance range and little consequence in the high-temperature/high-irradiance range. Translation to $T_{\text {ref }}$ and $J_{\text {sc,ref }}=19.3 \mathrm{mAcm}^{-2}$ results in the blue open diamonds. The curvature in the translated data $G_{\text {sc @Tref,Jsc,ref }}$ (open diamonds) in Fig. 4a) indicates that the simple linear dependence of $G_{\mathrm{sc}}$ on $J_{\text {sc,meas }}$ is not perfectly accurate. However, restriction of the range of analysis to higher values of $J_{\text {sc,meas }}\left(15 \mathrm{mAcm}^{-2} \cdot J_{\text {sc,meas }} \cdot 25 \mathrm{mAcm}^{-2}\right)$ yields a flat distribution of the translated data (blue full diamonds). Regardless of the fitting range, the major effect of bias-dependent current collection is accounted for by the use of Eqs. 10 and 11, especially when comparing the data in the early state (Fig. 4a), week $1, G_{\text {sc@ref }} \cdot 2.3 \mathrm{mScm}^{-2}$ ) with a later state of exposure (Fig. 4b), week 106, $\left.G_{\text {sc@ref }} \cdot 5.5 \mathrm{mScm}^{-2}\right)$. 


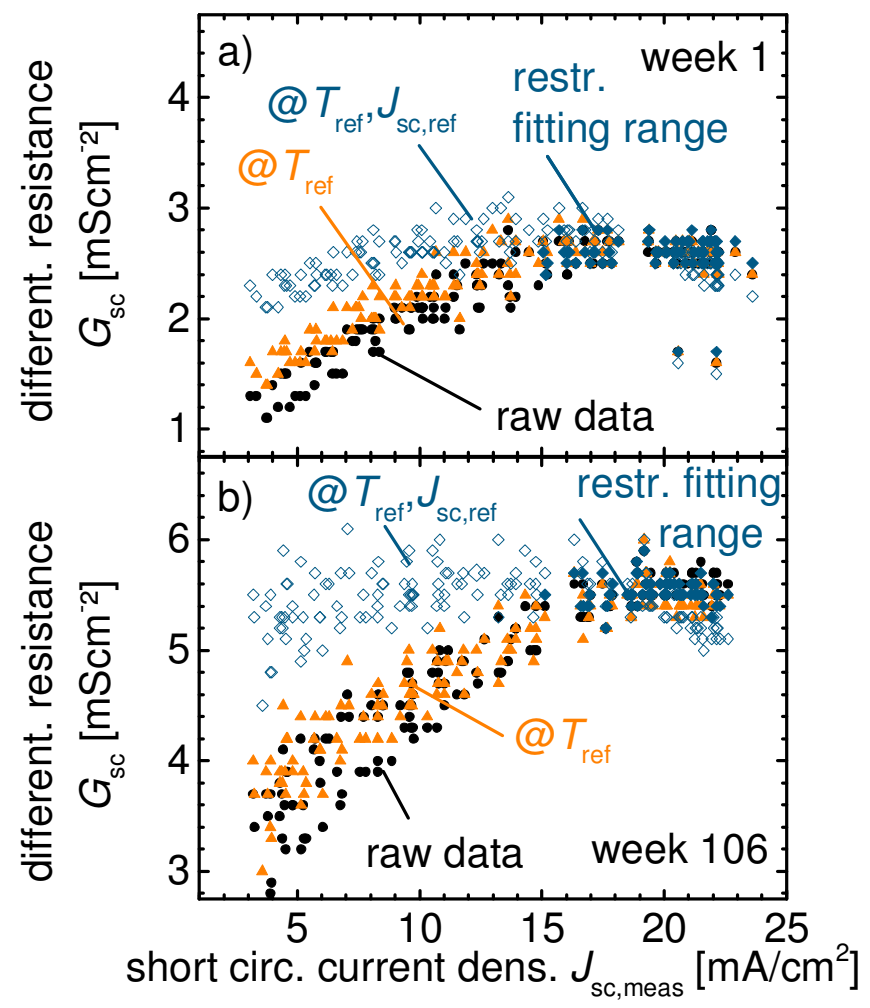

Fig. 4. Translation of differential conductance $G_{s c, \text { meas }}$ from outdoor-measured JV curves to reference conditions for week $1(a)$ and week $106(b)$. The raw data $G_{s c \text { meas }}$ (black dots) are translated to the reference temperature $T_{r e f}=40^{\circ} \mathrm{C}$ (full triangles), and additionally current density $J_{s c, \text { ref }}=19.3 \mathrm{mAcm}^{-2}$ (open diamonds) according to Eq.(11). Restricting the fitting range to current densities $15 \mathrm{mAcm}$ ${ }^{2} \leq J_{\text {sc,meas }} \leq 25 \mathrm{mAcm}^{-2}$ yields a flatter distribution of the corrected data (full diamonds).

Figure 5 depicts the coefficients obtained from fitting Eq. (10) to the weekly data intervals together with the value $G_{\text {scœref }}$ (black squares) translated to reference conditions. Note that here and in the Figs. 7, 9, 10, 11, and 12, the given data have been calculated using the restricted fitting range of short-circuit current densities $15 \mathrm{mAcm}^{-2} \cdot J_{\text {sc,meas }} \bullet 25 \mathrm{mAcm}^{-2}$. In Fig. 5a), the coefficient for the temperature correction $\alpha_{\mathrm{Gsc}}$ and the extrapolated shunt conductance $G_{\mathrm{sc}, 0}$ have been combined to $G_{\mathrm{sc}, 0 @ \mathrm{Tref}}=G_{\mathrm{sc}, 0}+\alpha_{\mathrm{Gsc}} T_{\text {ref }}$ (gray squares), i.e., the shunt conductance at reference temperature. Figure $5 b)$ gives the coefficient for current correction $\kappa_{\text {Gsc }}$. All values increase over time. The annual degradation of $G_{\text {sc. } 0 \oplus \text { Tref }}\left(0.62 \mathrm{mScm}^{-2} / y e a r\right)$ and that of $\kappa_{\text {Gsc }}$ multiplied by $J_{\text {ref }}\left(0.68 \mathrm{mScm}^{-2} /\right.$ year $)$ add up to the degradation of $G_{\text {sceref }}\left(1.3 \mathrm{mScm}^{-2} /\right.$ year $)$. Thus, the overall increase of $G_{\text {sc@ref }}$ is caused equally by the increase of the irradiance-independent shunt resistance and by the increasing influence of bias-dependent photocurrent collection. The latter effect is negligible at the beginning of the exposure, with $\kappa_{\text {Gsc }}$ being close to zero, but becomes more prominent during time of exposure, as already demonstrated by the data in Figs. 4a) and 4b). 


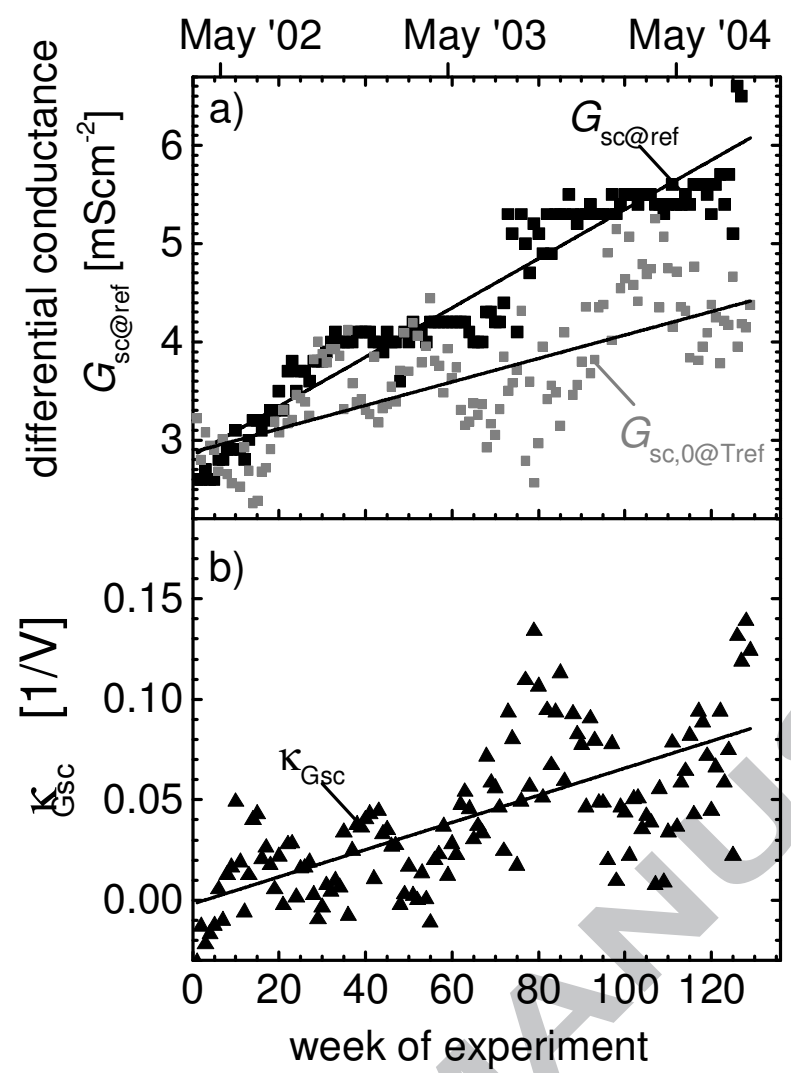

Fig. 5. Translation coefficients for $G_{s c}$. Coefficients $G_{s c, 0 @ T r e f}($ gray squares in $a), \kappa_{G s c}(b)$ gained from fitting the experimental data to Eq. (10) on a weekly basis, as well as the differential resistance $G_{s c @ r e f}$ (black squares in a) translated to the reference conditions $T_{r e f}=40^{\circ} \mathrm{C}_{\text {and }} \mathrm{J}_{\mathrm{sc}, \mathrm{ref}}=19.3 \mathrm{mAcm}^{-2}$ via Eq. (11).

\subsection{Open-circuit voltage}

The dependence of the open-circuit voltage $V_{\text {oc,meas }}$ on $T_{\text {meas }}$ and $J_{\text {sc,meas }}$ follows from the standard diode equation of an illuminated solar cell [Nelson, 2003]

$$
V_{\mathrm{oc}}=E_{\mathrm{a}} / q-n k T / q \log \left(J_{00}\right)+n k T / q \log \left(J_{\mathrm{sc}}\right) .
$$

Thus, we use the equation

$$
V_{\text {oc, meas }}\left(J_{\text {sc } \text { meas }}, T_{\text {meas }}\right)=V_{\text {oc }}^{0}-\left\{\alpha_{\text {Voc }}-\varepsilon_{\text {Voc }} \log \left(J_{\text {sc,meas }}\right)\right\} T_{\text {meas }} \text {. }
$$

The correction toward reference conditions is obtained via

$$
\begin{aligned}
& V_{\text {oc } @ \text { ref }}=V_{\text {oc, meas }}\left(J_{\text {sc, ref }}, T_{\text {ref }}\right)=V_{\text {oc }}^{0}-\alpha_{\mathrm{Voc}} T_{\text {ref }}+\varepsilon_{\mathrm{Voc}} T_{\text {ref }} \log \left(J_{\text {sc, ref }}\right) \\
& =V_{\mathrm{oc}, \text { meas }}-\left\{\alpha_{\mathrm{Voc}}-\varepsilon_{\mathrm{Voc}} \log \left(J_{\text {sc, meas }}\right)\right\}\left(T_{\text {ref }}-T_{\text {meas }}\right) \\
& +\varepsilon_{\mathrm{voc}} T_{\text {ref }} \log \left(J_{\mathrm{sc}, \text { ref }} / J_{\text {sc,meas }}\right)
\end{aligned}
$$

allowing again for the separate correction for $T_{\text {meas }}$ and $J_{\text {sc,meas }}$.

Figures 6a) and 6b) show the raw $V_{\text {oc }}$ data (black dots) and data translated to $T_{\text {ref }}$ (orange full triangles) for week 1 and 106, respectively. After elimination of the temperature dependence, the $V_{\text {oc }}\left[\log \left(J_{\text {sc }}\right)\right]$ relationship shows the linear (logarithmic) dependence expected from Eq. 13 (blue open diamonds). Also 
shown in the data is the fact that at low current densities, the slope of the semi-logarithmic plots (especially in Fig. 6b) is larger than in the range of large current densities. Such a "double-exponential" or "doublelogarithmic" behavior (of the $V_{\text {oc }}$ vs. $\log \left(J_{\text {sc }}\right)$ relationship) is known for most solar cells (see, e.g., Wolf et al., 1977). This behavior is due to different dominant recombination mechanisms at high and low bias. The diode ideality factor analyzed for current densities between 15 and $25 \mathrm{mAcm}^{-2}$ is slightly above unity, whereas the ideality factor analyzed for current densities between 4 and $7.4 \mathrm{mAcm}^{-2}$ is in the range of $3.5-$ 4.5. Such high ideality factors at low to moderate forward bias have been previously observed in early days of PV research (see, e.g., Queisser, 1962). A comprehensive review of different mechanisms leading to ideality factors larger than two was recently given by Breitenstein (2013). Note that models for doubleexponential behavior in the classical sense are one-dimensional models. However, spatially extended models (considering networks of resistors and diodes) also predict double-exponential behavior, including unusually high diode ideality factors [Grabitz et al., 2005; Breitenstein, 2013; Breitenstein and Rissland, 2013]. Thus, we must keep in mind that although most of our discussion is conducted in terms of simple one-dimensional models, the spatially extended, inhomogeneous nature of the module might offer different explanations.

Because of the double-exponential behavior, Eqs. (13) and (14) must be applied separately to different $J_{\text {sc }}$ ranges. At the beginning of exposure (Fig. 6a), the secondary diode is not very pronounced. Consequently, a translation of the measured values $V_{\text {oc,meas }}$ to the standard conditions $T_{\text {ref }}$ and $J_{\text {sc,ref }}$ leads to constant values $V_{\text {oc@ref }}$ in the entire range of short-circuit current densities (open blue diamonds). With increasing exposure time, the secondary diode becomes more pronounced (bending in Fig. 6b). Therefore, application of a single diode model for all values $J_{\text {sc,meas }}$, instead of yielding constant values, leads to a considerable bending of $V_{\text {oc@ref }}$ (open diamonds). In contrast, restriction of the fitting range to high bias conditions of $15 \mathrm{mAcm}^{-2} \cdot J_{\text {sc,meas }} \cdot 25 \mathrm{mAcm}^{-2}$ leads to constant values for $V_{\text {oc@ref }}$ (full diamonds). Thus, a selfconsistent transformation of the measured values is only feasible under restriction to bias conditions where the data correspond to a single diode, either the primary or secondary one.

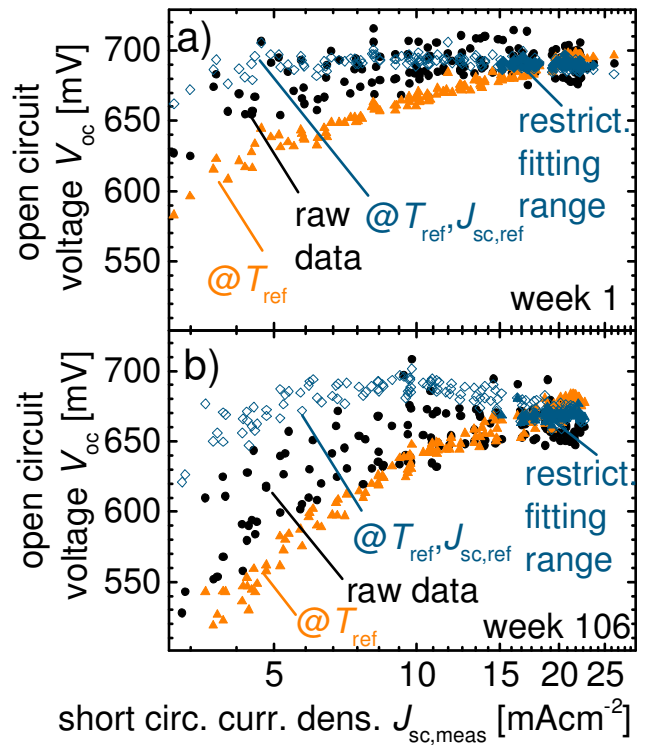

Fig. 6. Translation of open-circuit voltage $V_{\text {oc,meas }}$ from outdoor-measured JV curves to reference conditions $\left(T_{\text {ref }}=40^{\circ} \mathrm{C}\right.$ and $\left.J_{s c, r e f}=19.3 \mathrm{mAcm}^{-2}\right)$ for a) week 1 and $\left.b\right)$ week 106. The $V_{\text {oc,meas }}$ data extracted from the measured JV curves (black dots) are translated to reference temperature $T_{\text {ref }}$ (full triangles)and current 
density $J_{\text {sc,ref }}$ (open diamods). Restricting the fitting range to current densities $15 \mathrm{mAcm}^{-2} \leq J_{\text {sc,meas }} \leq 25$ $\mathrm{mAcm}^{-2}$ yields a flatter distribution of the corrected data (full diamonds).

Figure 7 depicts the temporal change in the correction coefficients $\alpha_{\mathrm{Voc}}$ (gray open squares), $\varepsilon_{\mathrm{Voc}}$ (red open squares), and $V_{\mathrm{oc}, 0}$ (green full circles) and of the calculated voltage at reference conditions $V_{\text {oc@ref }}$ (full squares). A significant decrease of $V_{\text {oc@ref }}$ by about $20 \mathrm{mV}$ within the time of exposure is observed. A linear fit to the time series $V_{\text {oc@ref }}(\mathrm{t})$ yields an average decline of $9.2 \mathrm{mV} /$ year (per cell), whereas the linear fits show increases and decreases by about the same amount in $\varepsilon_{V o c}(\mathrm{t}) \times 313 \mathrm{~K} \times \log \left(19.3\left[\mathrm{mAcm}^{-2}\right]\right), V_{\text {oc }, 0}$ and $\alpha_{\mathrm{voc}} \times 313 \mathrm{~K}$. The sum results in the decline of $V_{\text {oc@ref*}}$

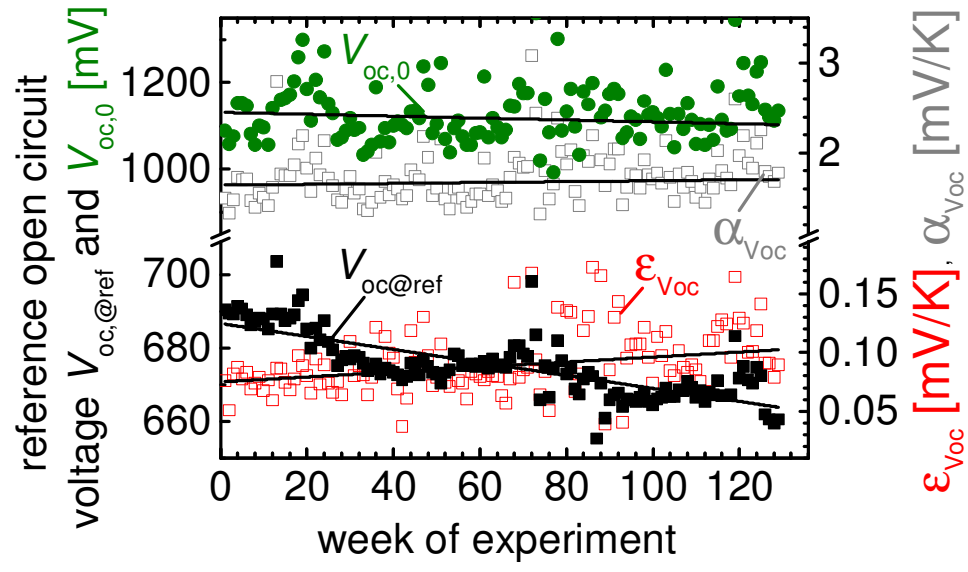

Fig. 7. Time evolution of $V_{\text {oc@ref }}$ and of the translation coefficients in Eq. (13) and Eq. (12). The linear fits to the coefficients $\alpha_{V o c}$ (gray open squares), $V_{o c, 0}$ (green dots), and $\mathcal{E}_{V_{\mathrm{oc}}}$ (red open squares) show that the contributions add up to the degradation of $V_{\text {oc@ref }}\left(\right.$ black dots) by almost equal shares. $V_{\text {oc@ref }}$ degrades by about 9.2( \pm 0.7$)$ mV/year.

\subsection{Differential resistance at open circuit}

The differential resistance $R_{\mathrm{oc}}$ at open circuit is a series connection of the diode resistance and a linear series resistance $R_{\mathrm{s}}$ according to

$$
R_{\text {oc, meas }}\left(J_{\text {sc,meas }}, T_{\text {meas }}\right)=R_{\mathrm{s}}+\beta_{\text {Roc }} T_{\text {meas }} / J_{\text {sc,meas }}+\alpha_{\text {Roc }} T_{\text {meas }} .
$$

Note for the diode resistance that the coefficient $\beta_{\text {Roc }}$ in a single-diode model corresponds to $n k / q$, where $k$ denotes Boltzmann's constant, $q$ the elementary charge, and $n$ the diode ideality factor. The equation used corresponds to Eq. 9 in Werner (1988). The correction function resulting from Eq. 15 finally reads

$$
\begin{aligned}
R_{\text {oc } @ \text { ref }}=R_{\text {oc, meas }} & -\beta_{\text {Roc }}\left(T_{\text {meas }} / J_{\text {sc, meas }}-T_{\text {ref }} / J_{\text {sc }, \text { ref }}\right) . \\
& -\alpha_{\text {Roc }}\left(T_{\text {meas }}-T_{\text {ref }}\right)
\end{aligned} .
$$




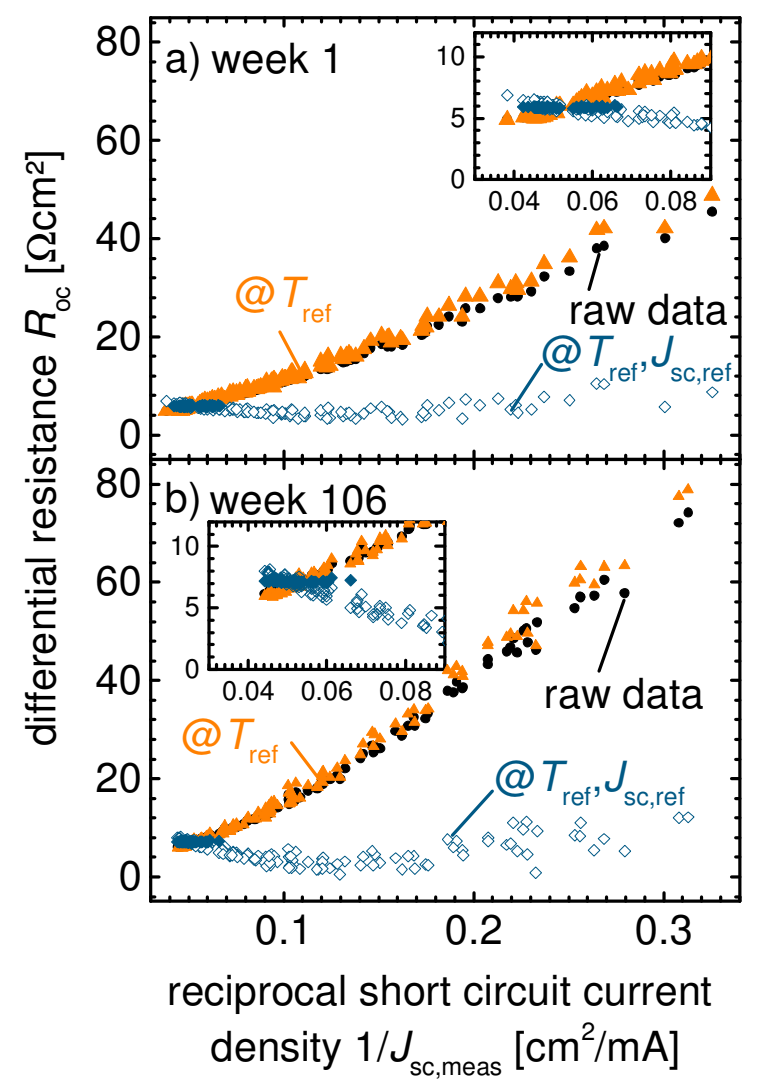

Fig. 8. Translation of open-circuit resistance $R_{o c}$ from outdoor-measured JV curves to reference condition. a) Week $1, b$ ) week 106, $R_{o c}$ extracted from the measured $J V$ curves (black dots) is translated to reference temperature $T_{\text {ref }}$ (orange full triangles). The translation to both temperature $T_{\text {ref }}$ and irradiance/current $J_{s c \text {,ref }}$ conditions (open diamonds) results in an almost straight line. Restricting the fitting range to current densities $15 \mathrm{mAcm}^{-2} \leq J_{s c \text {,meas }} \leq 25 \mathrm{mAcm}^{-2}$ yields a flatter distribution of the corrected data (full diamonds), as also highlighted in the insets in a) and b) that zoom into the high current-density range of the same data. 


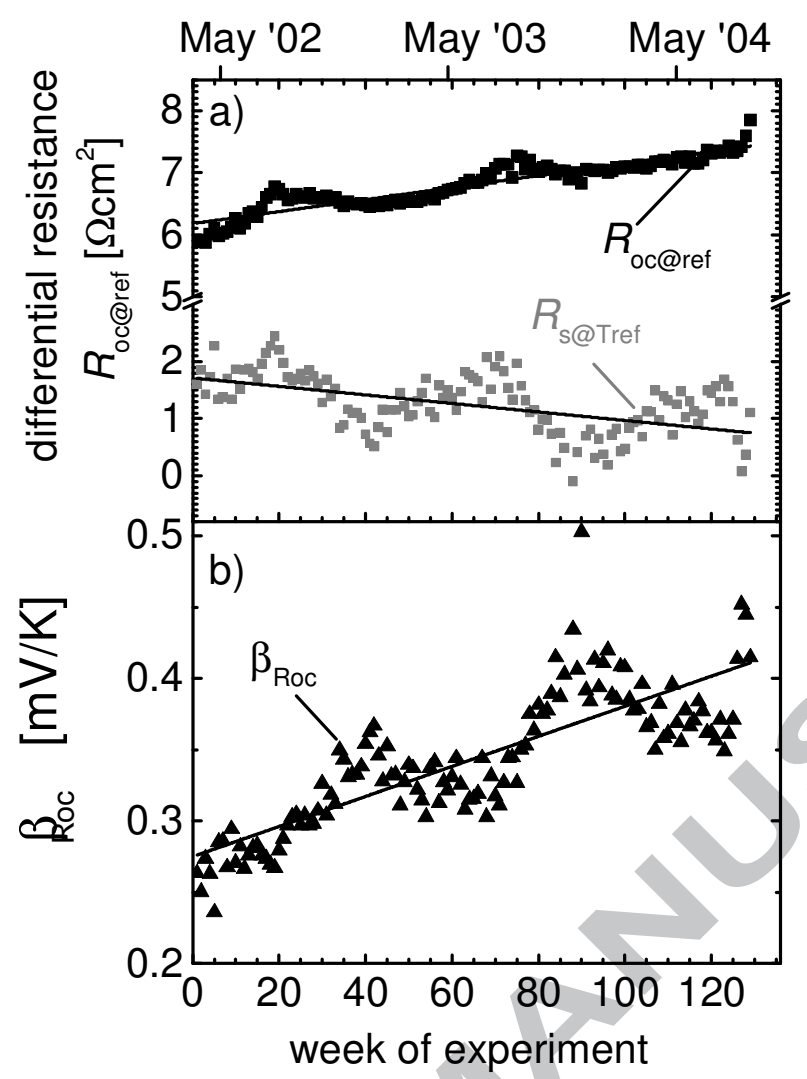

Fig. 9. Time evolution of $R_{o c @ r e f}\left(T_{\text {ref }}=40^{\circ} \mathrm{C}, J_{\text {sc,ref }}=19.3 \mathrm{mAcm}^{-2}\right)$ and of the translation coefficients. $R_{s @ T r e f}($ gray squares in $a)$ and $\beta_{R o c}(b)$ are gained from fitting the experimental data to Eq. (15) on a weekly basis. The linear fit to $R_{s @ r e f}$ reveals a decrease of $0.5 \Omega \mathrm{cm}^{2} / y e a r, \beta_{\text {Roc }}$ increases by $0.05 \mathrm{mV} / \mathrm{K} /$ year. The latter corresponds to an increase by $0.9 \Omega \mathrm{cm}^{2} / y e a r$, which adds up and is consistent with the increase in $R_{\text {oc@ref }}$ (black squares) of $0.5 \Omega \mathrm{cm}^{2} /$ year.

Figures $8 \mathrm{a}$ ) and $8 \mathrm{~b}$ ) display experimental $R_{\mathrm{oc}}$ data gained at different times of outdoor exposure: week 1 (Fig. 8a) and week 106 (Fig. 8b). The raw data (black dots) exhibit a drastic increase during those two years of outdoor operation. Temperature correction transforms the measured values $R_{\text {oc,meas }}$ to $R_{\text {oc@Tref }}$ (orange triangles) with only little change. At the beginning of exposure, the plot $R_{\text {oc@Tref }}$ vs. $1 / J_{\text {sc }}$ (Fig. 8a) yields approximately a straight line —as expected from the single-diode law underlying Eq. (15) whereas the corresponding data in Fig. 8b) exhibit two different slopes under high-bias and low-bias conditions. This finding is in agreement with the double-logarithmic feature found in the $V_{\mathrm{oc}} \mathrm{vs} . \log \left(J_{\mathrm{sc}}\right)$. Consequently, the final correction for both, temperature and current density, yields a clear bending in the data (open diamonds) Only restriction of the fitting range $15 \mathrm{mAcm}^{-2} \cdot J_{\text {sc,meas }} \cdot 25 \mathrm{mAcm}^{-2}$ (full diamonds), also highlighted in the inset, yields a flat distribution of the corrected data.

Figure 9a) displays the time evolution of the differential resistance $R_{\text {oc@ref }}$ (black squares), which shows a considerable increase of $0.5 \Omega \mathrm{cm}^{2} /$ year. The Ohmic series resistance $R_{\mathrm{s} @ \text { Tref }}=R_{\mathrm{s}}+\alpha_{\mathrm{Roc}} T_{\text {ref }}$ (gray squares) at reference temperature is relatively small (around $1-2 \Omega \mathrm{cm}^{2}$ as compared to $6-7 \Omega \mathrm{cm}^{2}$ for $R_{\text {oc } @ \mathrm{ref}}$ ). Because of Eq. (15), the increase of $R_{\text {oc@ref }}$ is defined by the sum of the decrease of $R_{\text {s@Tref }}$ by $0.5 \Omega \mathrm{cm}^{2} /$ year and the annual increase of $\beta_{\mathrm{Roc}}$ by $0.055 \mathrm{mV} / \mathrm{K} \times 313 \mathrm{~K} / 19.3 \mathrm{mAcm}^{-2}=0.9 \Omega \mathrm{cm}^{2}$. Note that the increase of $\beta_{\text {Roc }}$ is 
equivalent to an increase of the diode ideality factor $n$. This observation will be more closely discussed in sect. 5.2.

\section{Time Evolution of Photovoltaic Parameters}

\subsection{Degradation at standard conditions}

Up to this point, we have analyzed the four parameters of the $\mathrm{KH}$ equation individually with the help of the time evolution of physically motivated coefficients. The physical meaning of the four parameters opens up the opportunity to interpret the physics behind the degradation. This is not possible for most existing methods where the parameters relate to quantities that cannot directly be interpreted (as e.g. the $F F$ ). Because the fill factor depends on $R_{\mathrm{sc}}$ and $R_{\mathrm{oc}}$ via Eq. (2), we are now able to reconstruct the time evolution of the complete set of PV parameters from our results above. Figure 10 shows the time evolution of shortcircuit current density $J_{\text {sc@ref, }}$ open-circuit voltage $V_{\text {oc@ref }}$, and the power $P_{\text {mpp@ref }}$, and fill factor $F F_{@ \text { ref }}$ calculated from the $J V$ curves translated to reference conditions. The $F F_{@ \text { ref }}$ and $P_{\text {mpp@ref }}$ data were corrected after Eq. A.2. As stated earlier, $J_{\text {sc@ref }}$ fluctuates with seasonal spectral changes but without significant degradation $\left(<0.1 \%\right.$ /year) and $\mathrm{V}_{\text {ос @ref }}$ decreases by a rate of $9.2 \mathrm{mV}(1.3 \%)$ per year. However, the major influence on the degradation of the output power $\left(5.8 \%\right.$ /year) stems from the degradation of the $F F_{@ \text { ref }}(4.5 \% /$ year $)$.

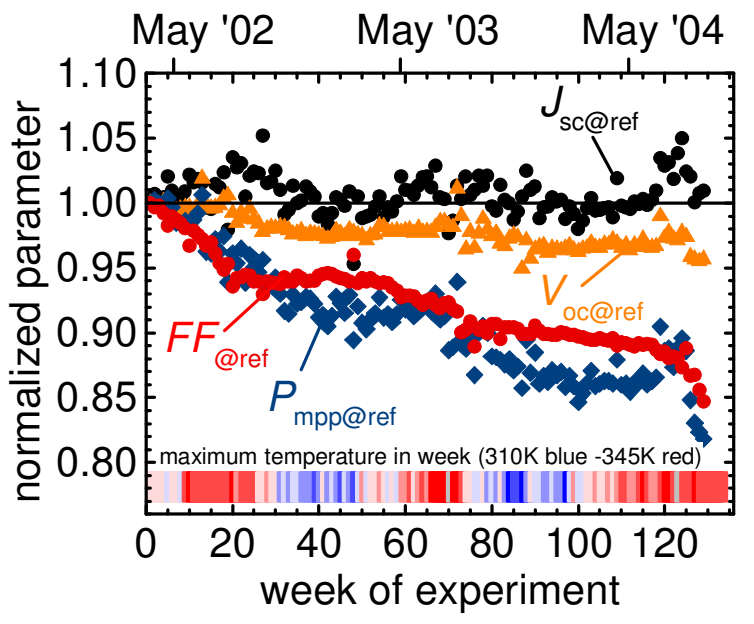

Fig. 10. Time evolution of the normalized PV parameters at standard reference conditions $\left(T_{r e f}=40^{\circ} \mathrm{C}\right.$, $\left.\phi_{\text {ref }}=1000 \mathrm{Wm}^{-2}\right)$. A degradation of the seasonally fluctuating short-circuit current density $J_{\text {sc@ref }}($ black dots) is not significant $(<0.1 \% / y e a r)$. The open-circuit voltage $V_{\text {oc@ref }}$ (orange triangles) degrades by about $1.3 \%$ per year. The degradation of the fill factor $F F_{@ \text { ref }}$ (red circles) by about $4.5 \%$ per year yields the largest contribution to the degradation of the maximum power output $P_{m p p @ r e f}(5.8 \% / y e a r$, blue diamonds).

The dependence of the fill factor $F F_{\text {@ref }}$ on $R_{\text {oc@ref }}$ and $R_{\text {sc@ref }}$ enables us to determine how much of the degradation is due to changes in each parameter. Figure 11 displays the original calculated fill factor data $F F_{\text {@ref }}$ normalized to the start value at week 1 (black dots, same data as in Fig. 10). All data were corrected as explained in A.1. Also shown are two sets of reconstructed $F F_{@ \text { ref }}$ data with the respective influence of $R_{\text {oc@ref }}$ and $R_{\text {sc@ref }}$ degradation removed. The difference from the original data to the data with $R_{\text {oc@ref }}$ degradation removed is slightly larger than the difference from the data with $R_{\text {sc@ref }}$ degradation removed. This finding points to an influence of $R_{\text {sc@ref }}$ degradation on $F F_{@ \text { ref }}$ degradation that is slightly larger than that 
of $R_{\text {oc@ref }}$ degradation. However, because both $R_{\text {oc@ref }}$ and $R_{\text {sc@ref }}$ degradation essentially result from the increasing influence of bias-dependent carrier collection, this mechanism is also the main source of the overall $F F_{@ \text { ref }}$ degradation.

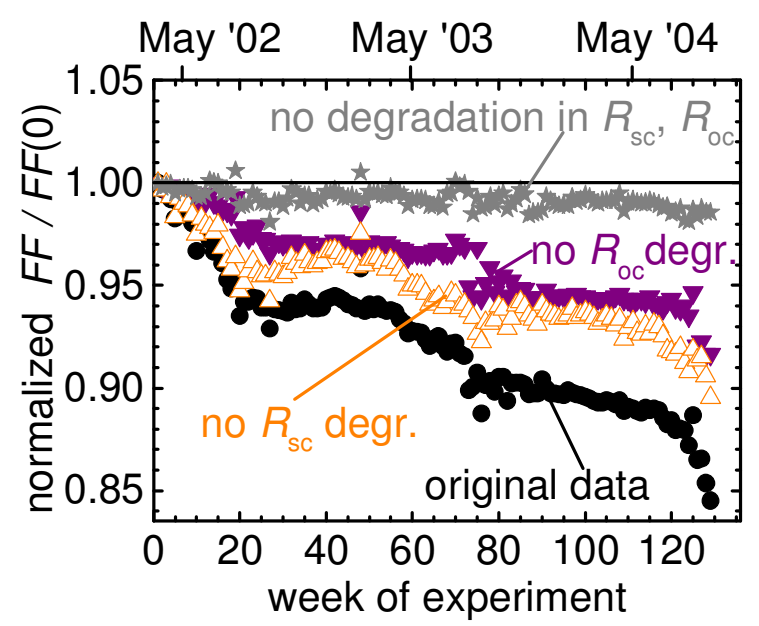

Fig. 11. Origin of fill factor degradation. The fill factor $F F_{@ r e f}$ drop of the JV curves was calculated via Eq. (2) and normalized to compare the influence of degradation in $R_{s c @ r e f}$ and $R_{\text {oc@ref }}$ combined (original data, black dots), only in $R_{s c @ r e f}\left(\right.$ purple full triangles), respectively, $R_{\text {oc@ref }}$ (orange open triangles), and no degradation in the two (gray stars). $R_{s c @ r e f}$ degradation dominates the FF@ref degradation slightly. Assuming no degradation in $R_{\text {oc@ref }}$ and $R_{\text {sc @ref; }}$; only a loss $<1 \%$ remains of the 10.6\% total FF@ref loss after 2.5 years of outdoor exposure.

\subsection{Influence of voltage-dependent photocurrent collection on the ideality factor}

The diode ideality factor $n$ can be calculated from the available data in two ways: from Eq. 12 (corresponding to a plot of $V_{\mathrm{oc}}$ vs. $\log \left(J_{\mathrm{sc}}\right)$, cf. Fig. 6), and from Eq. 16 (corresponding to a plot of $R_{\mathrm{oc}}$ vs. $1 / J_{\text {sc }}$, cf. Fig. 8). For a simple single-diode model, both methods should yield the same result. As shown in appendix A.2, the voltage-bias dependence of the photocurrent affects the $V_{\mathrm{oc}} \mathrm{vs} \log \left(J_{\mathrm{sc}}\right)$ and the $R_{\mathrm{sc}} \mathrm{vs}$. $1 / J_{\mathrm{sc}}$ relationship in different ways: The $V_{\mathrm{oc}}$ vs. $\log \left(J_{\mathrm{sc}}\right)$ method yields ideality factors $n_{\mathrm{voc}}$ that are lower than those of the single-diode model, as already shown by Eron and Rothwarf (1984). In contrast, the ideality factor $n_{\text {Roc }}$ obtained from the $R_{\text {sc }}$ vs. $1 / J_{\text {sc }}$ method substantially overestimates the ideality factor. Thus, a systematic discrepancy between the results of both methods represents a clear indication of voltagedependent carrier collection. Figure 12 compares the ideality factors determined by the two different methods. Note that this analysis is based on data under high irradiation conditions with corresponding shortcircuit current densities $15 \mathrm{mAcm}^{-2} \bullet J_{\text {sc,meas }} \cdot 25 \mathrm{mAcm}^{-2}$ to minimize the influence of the secondary diode on both results. Figure 12 demonstrates that $n_{\text {Roc }}$ exceeds $n_{\mathrm{Voc}}$ by a factor of 3-4 over the entire time of the experiment. Additionally, it is seen that the ideality factor $n_{\text {Roc }}$ increases by about 0.6 per year (from a linear fit) as compared to an increase of 0.13 for $n_{\mathrm{Voc}}$. These findings highlight the influence of bias-dependent carrier collection on the overall device behavior, as well as on the degradation of the module. We conclude

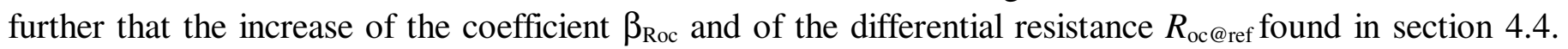
also result from the increasing influence of bias-dependent photocurrent collection. This mechanism is consequently the main source of the module degradation. 


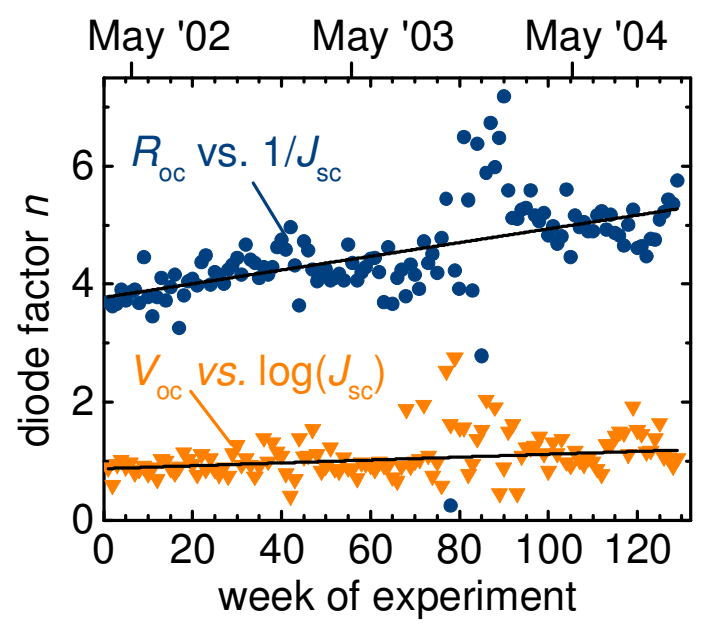

Fig. 12. Determination of the ideality factor $n$. Comparison of the ideality factors determined from the temperature-corrected data via the $R_{\mathrm{oc}}$ vs. $1 / J_{\mathrm{sc}} \operatorname{method}\left(n_{\text {Roc }}\right.$, blue dots) and via the $V_{\mathrm{oc}} v s . \log \left(J_{\mathrm{sc}}\right)$ method ( $n_{\mathrm{voc}}$ orange triangles). The ideality factor from $R_{\mathrm{oc}}$ vs. $1 / J_{\mathrm{sc}}$ exhibits an increase of 0.6/year (compared to $0.13 /$ year for $n_{V o c}$ ), indicating the increasing influence of bias-dependent current collection. The database for the analysis is restricted to short-circuit current densities $15 \mathrm{mAcm}^{-2} \leq J_{\text {sc,meas }} \leq 25 \mathrm{mAcm}^{-2}$.

\section{Model Comparison}

The focus of the present work is on the analysis of the degradation behavior of the CdTe module. However, based on the present analysis a prediction is also possible for the output power of the module at a given irradiance and module temperature. The following sketches some differences between our approach and established PV performance models from the literature [Sutterlueti et al. 2009, de Soto et al., 2006, Merten et al. 1998].

PV performance models are intended to describe the dependence on the maximum output power $P_{m p p}=P_{m p p}(\phi, T, .$.$) as a function of irradiance intensity \phi$, module temperature $T$, and possibly further variables characterizing the incident spectrum, like the average photon energy [Jardine et al., 2002]. Often these performance models fulfill a second scope, namely to analyze the behavior of $P_{m p p}(\phi, T, .$.$) in terms of$ parameters that are gained from the $J V$ characteristics of a module or a system. This is either obtained by fitting of the entire $J V$ characteristic to specific equation $J=J\left[V, p_{1}, p_{2}, \ldots\right]$ with a number $i=1, . ., N$ of parameters, as in the present case as well as in typical diode models [de Soto et al., 2006], or by extracting the parameters only at characteristic points of the curves (e.g., the Loss Factors Model, LFM [Sutterlueti et al., 2009]). In fitting models the functional dependence $P_{m p p}=P_{m p p}(\phi, T, .$.$) is indirectly obtained by re-$ evaluation of $J V$ curves. In the LFM the output power is a simple product of normalized parameters, a reference value, and correction functions for $\phi$ and $T$. Therefore, the LFM might de denoted as a factorization model.

In all models, the number $N$ of extracted parameters represents a compromise between the requirement for precision in predicting the performance on the one side and the requirement for a physical meaningful analysis on the other side. For the former purpose, a higher number $N$ increases the precision whereas for a physically meaningful analysis the number of independent parameters should be restricted to those parameters that have a significant dependence on the external variables such that one can describe the parameters with the help of dependencies $p_{i}(\phi, T, .)=.p_{i}\left(\phi, T, . ., c_{T}^{i}, c_{\phi}^{i}, ..\right)$ yielding a set of coefficients $\left(c_{T}^{i}, c_{\phi}^{\phi}, ..\right)$ 
for each parameter. The LFM contains $N=6$ and the dependence of each parameter on $\phi$ is described by three coefficients. The $T$ dependence of $J_{s c}$ and $V_{o c}$ in the LFM is linear whereas the other four parameters are assumed to be temperature independent. Single diode models use typically $N=5$, and in the present work we have restricted ourselves to $N=4$. This restriction allows us to put more emphasis on the specific analysis of the parameters. For instance, the dependence of $R_{s c}$ on $\phi$ or the discrepancy between ideality factors determined by different methods are unveiled by the present approach but are not covered by other approaches.

Finally all models predict the output power by its dependence on the parameters, i.e. $P_{m p p}=P_{m p p}\left(p_{1}\left(\phi, T, \ldots, c_{T}^{1}, c_{\phi}^{1}, ..\right), \ldots.\right)$. In fitting models the maximum output power is not a parameter (as it is in the LFM) but has to be recalculated from the $J V$ parameters using the fitting equation. Because of this fact and due to the restricted number of parameters, fitting models can introduce systematic errors. As shown in appendix A1, a systematic error of appr. $-0.3 \%$ at $30 \mathrm{Wm}^{-2}$ and $+1.0 \%$ at $100 \mathrm{Wm}^{-2}$ is introduced by the reconstruction of the $J V$ curves with the $\mathrm{KH}$ equation. Note that our degradation analysis (Figs. 10 and 11 ) is only marginally affected by this error because all values at all times and all temperatures are subject to the same error. However, a correction is required for the purpose of output power prediction. A simple correction function reduces the error to approx. $\pm 0.1 \%$ which is comparable to standard deviations in the predictions of other models (Stein et al., 2013). In contrast, the single diode model [de Soto et al., 2006], though having 5 parameters compared to the 4 four parameters of the $\mathrm{KH}$ equation, introduces a larger systematic error of $3 \%$ and a larger standard deviation of $\mathrm{xx} \%$ compared to the $\mathrm{KH}$ equation for the present data set. As shown in Appendix A.1 our correction procedure can eliminate the systematic error also for the diode model. However, the standard deviation after correction is still four times larger than for the corrected data based on the KH equation.

\section{Conclusions}

The application of the four-parameter Karmalkar-Haneefa $(\mathrm{KH})$ equation enables one to narrow down the analysis of outdoor-acquired $J V$ data to a minimum set of four parameters. Appendix A1 shows that this fitting is much more accurate than fitting the same data set to a five parameter diode equation [de Soto et al., 2006]. For the subsequent degradation analysis, the dependence of each of the four parameters on irradiation intensity and module temperature $\left(J_{\mathrm{sc}}\right)$ or on short-circuit current density and module temperature $\left(R_{\mathrm{sc}}, V_{\mathrm{oc}}\right.$, and $\left.R_{\mathrm{oc}}\right)$ is described by standard device-physics models and instead of norm IEC60891 used to translate the data to standard conditions. The dependence of the model coefficients on outdoor exposure time allows one to identify mechanisms underlying the time evolution of the parameters $J_{\mathrm{sc}}, R_{\mathrm{sc}}, V_{\mathrm{oc}}$, and $R_{\mathrm{oc}}$ at reference conditions. Because the fill factor is determined unambiguously by the four parameters, we can also reconstruct on the same basis the time evolution of the output power and the efficiency of the module, thereby discriminating the specific effects of the individual parameters and their coefficients. The accuracy of this prediction is increased by a simple linear correction where the coefficients are extracted directly from the raw data. For the case of the present CdTe module, we have shown that a mechanism with the same indicators as bias-dependent current collection has a major influence on the differential resistances $R_{\mathrm{sc}}$ and $R_{\mathrm{oc}}$ at short and open circuit. This mechanism is observable at the beginning of outdoor exposure, but also dominates the degradation behavior. This is demonstrated by the fact that the degradation of $R_{\mathrm{sc}}$ and $R_{\mathrm{oc}}$, which causes the degradation of the fill factor, is dominated by the same mechanism. Finally, we must keep in mind that effects arising from the spatially distributed nature of a solar module with large areas connected in parallel and many cells connected in series will certainly impact the behavior of the analyzed 
parameters. Therefore, we expect that future analyses based on spatially resolved models for thin-film modules will offer refined explanations for some of the observed features.

\section{Acknowledgments}

This work was funded by SO-075 'Helmhotz-NREL Solar Energy Initiative' (HNSEI). Financial support by the German Federal Ministry for the Environment, Nature Conservation, Building and Nuclear Safety ('PV Klima,' project number 0325517C) is also acknowledged. Furthermore, this work was also supported by the U.S. Department of Energy under Contract No. DE-AC36-08-GO28308 with the National Renewable Energy Laboratory. The authors give special thanks to David Albin (NREL) for valuable discussions.

\section{REFERENCES}

Bowden, S., Rohatgi. A., 2001. Rapid and accurate determination of series resistance and fill factor losses in industrial silicon solar cells. Proc. $17^{\text {th }}$ EUPVSEC, 1802-1806.

Breitenstein, O., 2013. Understanding the current-voltage characteristics of industrial crystalline silicon solar cells by considering inhomogeneous current distributions. Opto-Electronics Reviews 21, $259-282$.

Breitenstein, O., Rissland, S., 2013, A two-diode model regarding the distributed series resistance. Sol. Ener. Mat. and Sol. Cells 110, 77-86.

Carlsson, T., Brinkman, A., 2006. Identification of degradation mechanisms in field-tested CdTe modules. Prog. Photovolt: Res. Appl. 14(3), 213-244.

Corwine, C. R., Pudov, A. O., Gloeckler, M., Demtsu, S. H., Sites, J. R., 2004. Copper inclusion and migration from the back contact in CdTe solar cells. Sol. Ener. Mat. and Sol. Cells 82(4), 481-489.

Cotfas, D. T., Cotfas, P. A., Kaplanis, S., 2013. Methods to determine the dc parameters of solar cells: a critical review. Renew. and Sustain. Ener. Rev. 28, 588-596.

del Cueto, J. A., 2005. Closed-form solutions and parameterization of the problem of current-voltage performance of polycrystalline photovoltaic modules deployed at fixed latitude tilt, Photovoltaic Specialists Conference (PVSC), $31^{\text {st }}$ IEEE, 331-335.

del Cueto, J. A., von Roedern, B., 2006. Long-term transient and metastable effects in cadmium telluride photovoltaic modules. Prog. Photovolt: Res. Appl. 14(7), 615-628.

Das, A. K., Karmalkar, S., 2011. Analytical derivation of the closed-form power law J-V model of an illuminated solar cell from the physics based implicit model. IEEE Trans. on Electron. Dev. 58, 11761181.

EPIA Market Report 2013, published online, March 2014.

Eron, M., Rothwarf, A., 1984. Effects of a voltage-dependent light-generated current on solar cell measurements: $\mathrm{CuInSe} / \mathrm{Cd}(\mathrm{Zn}) \mathrm{S}$. Appl. Phys. Lett. 44, 131-133.

Guérin de Montgareuil, A., 2007. Description of MotherPV, the new method developed at INES / CEA for the assessment of the energy production of photovoltaic modules, Proc. $22^{\text {th }}$ EUPVSEC, 2608-2612. 
Gostein, M., Dunn, L., 2011. Light soaking effects on photovoltaic modules: overview and literature review. Photovoltaic Specialists Conference (PVSC), 37 ${ }^{\text {th }}$ IEEE, 003126-003131.

Grabitz, P. O., Rau, U., Werner, J. H., 2005. Modeling of spatially inhomogeneous solar cells by a multidiode approach. Phys. Stat. Sol. (a) 202, 2920-2927.

Hegedus, S. S., Desai, D., Thompson, C., 2007. Voltage dependent photocurrent collection in CdTe/CdS solar cells. Prog. Photovolt.: Res. Appl. 15, 587-602.

Hegedus, S. S., McCandless B. E., 2005. CdTe contacts for CdTe/CdS solar cells: effect of Cu thickness, surface preparation and recontacting on device performance and stability. Sol. Ener. Mat. and Sol. Cells 88(1), 75-95.

International Electrotechnical Commission 60891 ed.2.0, 2009. Photovoltaic devices - Procedures for temperature and irradiance corrections to measured I-V characteristics.

International Electrotechnical Commission 60904-1 ed.2.0, 2006. Photovoltaic devices - Part 1: Measurement of photovoltaic current-voltage characteristics.

Jardine, C. N., Gottschalg, R., Betts, T. R., Infield, D. G., 2002. Influence of spectral effects on the performance of multijunction amorphous silicon cells. Proceedings Photovoltaic in Europe Conference.

Jordan, D. C., Kurtz, S. R., 2014. The dark horse of evaluating long-term field performance—data filtering. IEEE J. Photovoltaics 4(1), 317-323.

Jenkins, C., Pudov, A., Gloeckler, M., Demtsu, S., Nagle, T., Fahrenbruch, A., Sites, J., 2003. CdTe back contact: response to copper addition and out-diffusion. NCPV and Solar Program Review Meeting.

Karmalkar, S., Haneefa, S., 2008. A physically based explicit J-V model of a solar cell for simple design calculations. IEEE Electron. Dev. Lett. 29, 449-451.

King, D.L., Boyson, W. E., Kratochvill, J. A., 2004. Photovoltaic Array Performance Model. Sandia Report, Sandia National Laboratories, Albuquerque, New Mexico, US.

Merten, J., Asensi, J.M., Voz, C., Shah, A.V., Platz, R., Andreu, J., 1998, Improved equivalent circuit and analytical model for amorphous silicon solar cells and modules. IEEE Trans. on Electron. Dev. 45, 423429.

Merten, J., Razongles, G., Sicot, L., Martin, J.-L., Beccavin, J., Villaume, J., Brock, C., 2012. Fast and precise PV module performance prediction from simple indoor measurements, Proc. $27^{\text {th }}$ EUPVSEC, 3302-3305.

Nelson, J., 2003. The Physics of Solar Cells, Imperial College Press, London.

Pudov, A. O., Gloeckler, M., Demtsu, S. H., Sites, J. R., Barth, K. L., Enzenroth, R. A., Sampath, W. S., 2002. Effect of back-contact copper concentration on CdTe cell operation. Photovoltaic Specialists Conference (PVSC), 29 ${ }^{\text {th }}$ IEEE, 760-763.

Queisser, H. J., 1962. Forward characteristics and efficiencies of silicon solar cells. Solid-St. Electron. 5, 110.

Sasala, R. A., Sites, J. R., 1993. Time dependent voltage in CuInSe ${ }_{2}$ and CdTe solar cells. Photovoltaic Specialists Conference (PVSC), $23^{\text {rd }}$ IEEE, 543-548.

De Soto, W., Klein, S.A., Beckman, W.A., 2006, Improvement and validation of a model for photovoltaic array performance. Solar Energy 80 78-88. 
Stein, J. S., Sutterlueti, J., Ransome, S., Hansen, C. W., King, B. H., 2013. Outdoor performance evaluation of three different models: single-diode, SAPM and Loss Factor Model. Proc. 28th EUPVSEC, 28652871.

Sutterlueti, J., Ransome, S., Kravets, R., Schreier, L., 2011. Characterising PV modules under outdoor conditions: what's most important for energy yield. Proc. $26^{\text {th }}$ EUPVSEC, 3608-3614.

Werner, J. H., 1998. Schottky barrier and pn-junction I/V plots- small signal evaluation. Appl. Phys. A 47, 291-300.

Williams, S. R., Betts, T. R., Gottschalg, R., Infield, D. G., Van der Borg, N. J. C. M., Burgers, A. R., De Moor, H., Warta, W., Friesen, G., Chianese, D., Guerin de Montgareuil, A., Zdanowicz, T., Stellbogen, D., Hermann, W., Pietruzko, S., Krustok, J., Dunlop, E., 2005. Evaluating the state of the art of photovoltaic performance modelling in Europe. Proc. 20 ${ }^{\text {th }}$ EUPVSEC, 1937-1941.

Wolf, M., Noel, G. T., Stirn, J., 1977, Investigation of the double exponential in the current-voltage characteristics of silicon solar cells. IEEE Trans. Electron. Devices, 24-ED, 419-428. 


\section{Appendix}

A.1.

The approximation by the $\mathrm{KH}$ equation fit the experimental data over a wide range of irradiances and temperatures reasonably well. However, it is advisable to investigate the error introduced into the reconstruction of the maximum power $P_{\text {mpp }}$ by reducing the number of parameters to four. Figure A.1a) plots the ratio $P_{\mathrm{mpp}, \text { raw }} / P_{\mathrm{mpp} \text {,fit }}$ of raw data to the data reconstructed from the $\mathrm{KH}$ equation as the function $P_{\mathrm{mpp}, \mathrm{fit}}$. The values of $P_{\text {mpp,raw }} / P_{\text {mpp,fit }}$ lie in a range of ca. +1.0 and $-0.5 \%$. A further reduction of this error is achieved by considering the linear relationship

$$
\frac{P_{\text {mpp,raw }}}{P_{\text {mpp,fit }}}=a+b P_{\text {mpp,fit }} .
$$

valid in the range $30 \mathrm{~W} / \mathrm{m}^{2}<P_{\text {mpp,fit }}<90 \mathrm{~W} / \mathrm{m}^{2}$ (Fig. A1). It is important to mention that this relationship is independent of exposure time and only implicitly dependent on irradiation intensity and module temperature. Thus, we can fit the entire data set by Eq. (A1) to obtain $a=0.9927$ and $b=0.017 \mathrm{~m}^{2} / \mathrm{W}$. In turn, the use of the correction equation

$$
P_{\mathrm{mpp}, \mathrm{corr}}=a P_{\mathrm{mpp}, \mathrm{fit}}+b P_{\mathrm{mpp}, \mathrm{fit}}^{2} .
$$

allows us to correct the reconstructed data and finally to reduce the root mean square standard deviation to +/- $0.085 \%$ (Fig. A1). Thus, we can maintain the convenience to reduce the JV-curves to four parameters with their dependence on irradiation intensity and module temperature and at the same time increase the accuracy of our prediction by a simple implicit correction.

To compare the systematic errors due to curve fitting between the $\mathrm{KH}$ equation and the single diode equation [de Soto et al., 2006] we fitted our entire data set also to

$$
J=J_{0}\left[\exp \left(\frac{q\left(V-J R_{s}\right)}{n_{i d} k T}\right)-1\right]+R_{p}\left(V-J R_{s}\right),
$$

where $J_{0}$ denotes the diode saturation current density, $R_{\mathrm{s}}$ the series resistance, and $R_{\mathrm{p}}$ the parallel resistance. Figure A1 b) demonstrates that the reconstructed maximum output power $P_{\text {mpp,fit }}$ differs by about $3 \%$ (average value of $P_{\mathrm{mpp}, \text { raw }} / P_{\mathrm{mpp}, \mathrm{fit}}$ is $97.13 \%$ for a range $30 \mathrm{~W} / \mathrm{m}^{2}<P_{\mathrm{mpp}, \mathrm{fit}}<90 \mathrm{~W} / \mathrm{m}^{2}$ ) due to the difficulties in modeling the shape of the curve. Obviously, the diode equation does not fit well the low-fill factor data of this thin-film CdTe module and the deviations between measured power and modeled power are significant. This systematic error is removed when applying the correction given by Eq. (A2). However, the great scatter in the data leads to a standard deviation of $0.329 \%$ which is about four times larger than the standard deviation of $0.085 \%$ resulting from the use of the $\mathrm{KH}$ equation. Thus, for the present module the use of the $\mathrm{KH}$ equation is more suitable for an accurate representation of the measured data than the conventional single diode equation. 


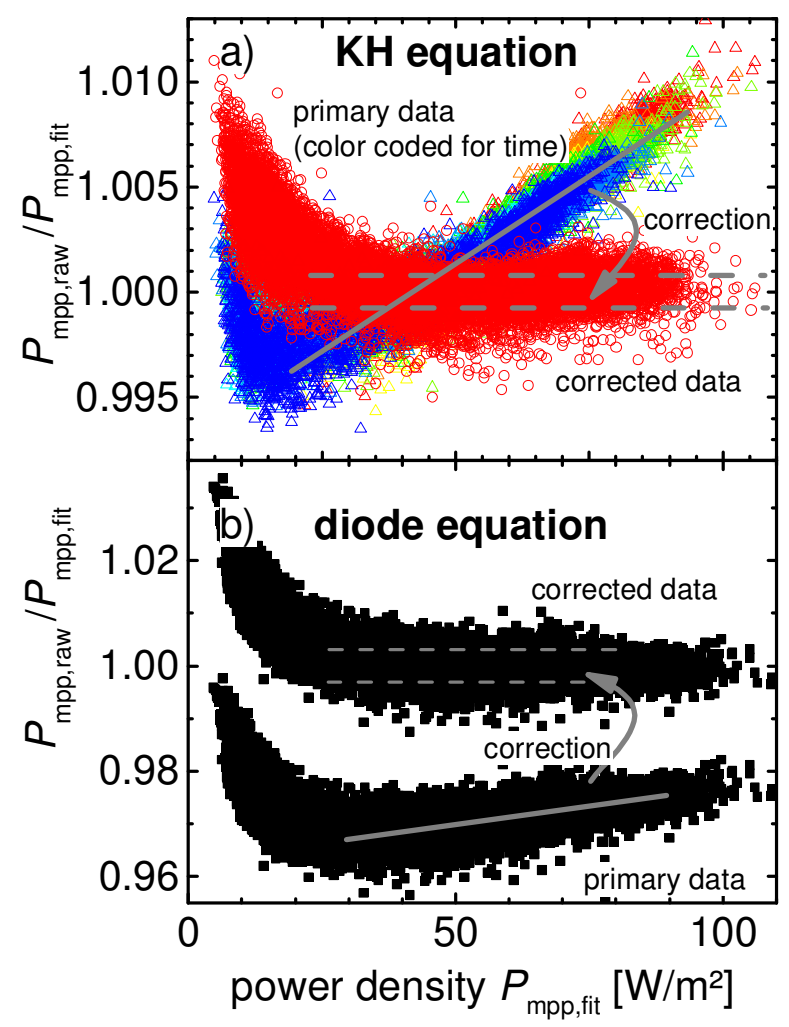

Fig. A1. Quotient of the power densities $P_{m p p, r a w}$ and $P_{m p p, f i t}$ of the raw and the fitted data (triangles). The data is color-coded for time (from red turning to yellow, green, and blue with increasing exposure time). a) The systematic error in the primary fit data (KH equation) is fitted linearly (solid line, Eq. (A1)) and the coefficients of the fits are used to gain the corrected data (red circles) via Eq. (A2) with a standard deviation of $0.085 \%$ (dashed lines). b) Quotient of the power densities $P_{\text {mpp,raw }} / P_{\text {mpp,fit }}$ for JV curves reconstructed by the single diode equation. The large systematic deviation of $3 \%$ is removed by the correction via Eq. (A2) whereas the remaining standard deviation amounts to 0.329\%, i.e., 3.88 times more than for the reconstruction via the KH equation. Note the difference in scale between a) and $b$ ).

A 2.

Voltage-bias dependent collection influences the value of $V_{\text {oc }}$ and the slope $R_{\text {oc }}$ in different ways. Therefore, the determination of the diode ideality factor $n$ via the $V_{\mathrm{oc}}\left(\log \left(J_{\mathrm{sc}}\right)\right)$ and via the $R_{\mathrm{oc}}\left(1 / J_{\mathrm{sc}}\right)$ relationship leads to different results.

Let us describe the $J V$ characteristics by its linear dependence of the current-collection function $f$ on the junction voltage $V_{\mathrm{j}}$ by

$$
J_{\mathrm{ph}}=J_{\mathrm{ph}}^{\max } f\left(V_{\mathrm{j}}\right)=J_{\mathrm{ph}}^{\max }\left[f_{\mathrm{sc}}+\Delta f-\chi\left(V-J R_{\mathrm{s}}\right)\right] .
$$


Here, $J_{\mathrm{ph}}^{\max }$ is the maximum photo-generated current density (at $f=1$ ), $f_{\mathrm{sc}}$ is the value of the collection function at short circuit, $f_{\mathrm{sc}}+\Delta f$ is the extrapolated value (see Fig. A2), and $\chi=d f / d V_{\mathrm{j}}$ is the slope of the collection function at $V_{\mathrm{j}}=V_{\mathrm{oc}}$.

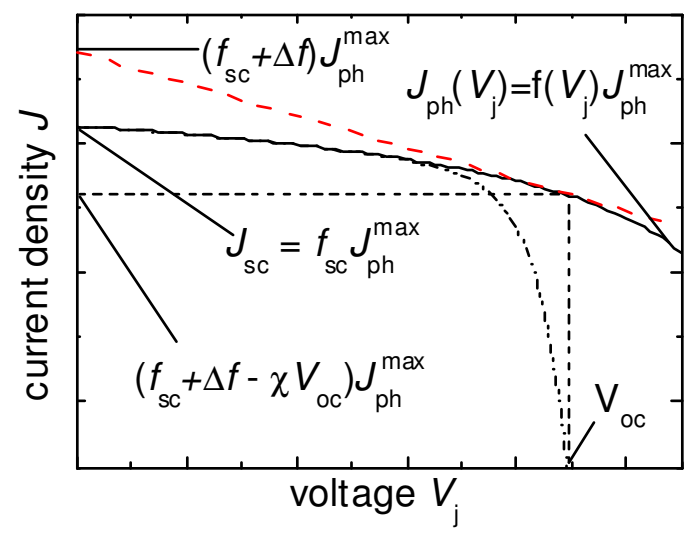

Fig. A2. JV characteristics (dash dotted line) described by its linear dependence on the current collection function $f$ on the junction voltage $V_{j} . J_{p h}\left(V_{j}\right)=f\left(V_{j}\right) J_{p h}{ }^{\max }$ according to Eq. Al (black line). The term $f_{S C}$ gives the value of the current-collection function at short-circuit conditions $J_{s c}, f_{S C}+\Delta f$ is the extrapolated value (red dashed line), and $\chi=d f / d V_{j}$ is the slope of the collection function at $V_{j}=V_{o c}$ (black dashed line marks $\left.V_{o c}\right)$.

Combining Eq. A4 with a conventional diode model yields

$$
\begin{aligned}
& J=J_{p h}^{\max }\left[f_{s c}+\Delta f-\chi\left(V-J R_{s}\right)\right]-J_{00} \exp \left(\frac{-E_{a}+q\left(V-J R_{s}\right)}{n k T}\right) \\
& =J_{s c}\left[1+\frac{\Delta f}{f_{s c}}-\frac{\chi}{f_{s c}}\left(V-J R_{s}\right)\right]-J_{00} \exp \left(\frac{-E_{a}+q\left(V-J R_{s}\right)}{n k T}\right)
\end{aligned}
$$

where the junction voltage $V_{\mathrm{j}}=V-J R_{\mathrm{s}}$ is calculated from the externally applied voltage $V$. At open circuit, it follows from Eq. A5

$$
J_{\mathrm{sc}}=J_{00} \frac{\exp \left(\frac{-E_{\mathrm{a}}+q V_{\mathrm{oc}}}{n k T}\right)}{1+\frac{\Delta f}{f_{\mathrm{sc}}}-\frac{\chi}{f_{\mathrm{sc}}} V_{\mathrm{oc}}}
$$

The ideality factor is then obtained from the logarithmic slope of the $V_{\mathrm{oc}}\left(\log \left(J_{\mathrm{sc}}\right)\right)$ relation according to 


$$
\frac{1}{n_{\mathrm{Voc}}}=\frac{k T}{q} \frac{d}{d V_{\mathrm{oc}}} \log \left(J_{\mathrm{sc}}\right)=\frac{1}{n}+\frac{1}{1+\frac{\Delta f}{f_{\mathrm{sc}}}-\frac{\chi}{f_{\mathrm{sc}}} V_{\mathrm{oc}}} \frac{k T}{q} \frac{\chi}{f_{\mathrm{sc}}}=\frac{1}{n}+\frac{J_{\mathrm{sc}}}{J_{\mathrm{ph}}\left(V_{\mathrm{oc}}\right)} \frac{k T}{q} \frac{\chi}{f_{\mathrm{sc}}}
$$

where $J_{\mathrm{ph}}\left(V_{\mathrm{oc}}\right)$ is the photocurrent density at $V_{\mathrm{oc}}$. Eq. (A4) implies that the ideality factor $n_{\mathrm{Voc}}$ obtained from this method overestimates the real diode ideality factor $n_{\mathrm{id}}$. However, this failure is relatively small, on the order of tenths of a percent [Eron and Rothwarf, 1984], because usually it holds that $f_{\mathrm{sc}} / \chi>>k T / q$.

The derivation of Eq. A5 with respect to the external voltage $V$ leads to

$$
G=\frac{d J}{d V}=\left\{J_{\mathrm{sc}} \frac{\chi}{f_{\mathrm{sc}}}-\frac{J_{00}}{n k T / q} \exp \left(\frac{-E_{\mathrm{a}}+q\left(V-J R_{\mathrm{s}}\right)}{n k T}\right)\right\}\left(1-G R_{\mathrm{s}}\right) .
$$

In the open-circuit situation, we have $J=0, V_{\mathrm{j}}=V=V_{\mathrm{oc}}$, and consequently

$$
G_{\mathrm{oc}}=J_{\mathrm{sc}}\left\{\frac{\chi}{f_{\mathrm{sc}}}-\frac{1}{n_{\mathrm{id}} k T / q}\left(1+\frac{\Delta f}{f_{\mathrm{sc}}}-\frac{\chi}{f_{\mathrm{sc}}} V_{\mathrm{oc}}\right)\right\}\left(1-G_{\mathrm{oc}} R_{\mathrm{s}}\right)
$$

Rearranging Eq. A9 via

leads to

$$
\begin{aligned}
& G_{\mathrm{oc}}\left(1+R_{\mathrm{s}} J_{\mathrm{sc}}\{\cdot\}\right)=J_{\mathrm{sc}}\{\cdot\}, \\
& G_{\mathrm{oc}}=J_{\mathrm{sc}}\{\cdot\} /\left(1+R_{\mathrm{s}} J_{\mathrm{sc}}\{\cdot\}\right), \\
& R_{\mathrm{oc}}=1 /\left(J_{\mathrm{sc}}\{\cdot\}\right)+R_{\mathrm{s}}
\end{aligned}
$$

$$
R_{\mathrm{oc}}=\frac{n k T / q}{J_{\mathrm{sc}}\left\{1+\frac{\Delta f}{f_{\mathrm{sc}}}-\frac{\chi}{f_{\mathrm{sc}}}\left[V_{\mathrm{oc}}+n k T / q\right]\right\}}+R_{\mathrm{s}} .
$$

Because $V_{\mathrm{oc}} \gg n k T / q$ and $J_{\mathrm{ph}}\left(V_{\mathrm{oc}}\right)=J_{\mathrm{ph}}^{\max }\left[f_{\mathrm{sc}}+\Delta f-\chi V_{\mathrm{oc}}\right]$, we can rewrite Eq. A13 by

$$
R_{\mathrm{oc}} \approx \frac{n k T / q}{J_{\mathrm{sc}}\left\{1+\frac{\Delta f}{f_{\mathrm{sc}}}-\frac{\chi}{f_{\mathrm{sc}}} V_{\mathrm{oc}}\right\}}+R_{\mathrm{s}}=\frac{n k T / q}{J_{\mathrm{ph}}\left(V_{\mathrm{oc}}\right)}+R_{\mathrm{s}}
$$

and the ideality factor determined via the $R_{\mathrm{oc}}\left(1 / J_{\mathrm{sc}}\right)$ relationship is given by

$$
n_{\mathrm{Roc}}=\frac{n}{\left\{1+\frac{\Delta f}{f_{\mathrm{sc}}}-\frac{\chi}{f_{\mathrm{sc}}} V_{\mathrm{oc}}\right\}}
$$


Likewise, we may write $n_{\mathrm{Roc}} / n=J_{\mathrm{sc}} / J_{\mathrm{ph}}\left(V_{\mathrm{oc}}\right)$. Thus, the $R_{\mathrm{oc}}\left(1 / J_{\mathrm{sc}}\right)$ method considerably overestimates the ideality factor. Typical values of the ratio $n_{\mathrm{Roc}} / n_{V o c} \approx n_{\mathrm{Roc}} / n$ in Fig. 15 amount to a range of 3-4, implying that only $25 \%-35 \%$ of the short-circuit current density is collected under open-circuit conditions. 